\title{
Duality and nonlinear response for quantum Hall systems
}

\author{
C. P. Burgess ${ }^{1,2, *}$ and Brian P. Dolan ${ }^{3, \dagger}$ \\ ${ }^{1}$ Institute for Advanced Study, Princeton, New Jersey 08540 \\ ${ }^{2}$ Physics Department, McGill University, 3600 University Street, Montréal, Québec, Canada H3A 2T8 \\ ${ }^{3}$ Department of Mathematical Physics, National University of Ireland, Maynooth, Ireland
}

(Received 6 June 2001; published 5 April 2002)

\begin{abstract}
We derive the implications of particle-vortex duality for the electromagnetic response of quantum Hall systems beyond the linear-response regime. This provides a theoretical explanation of the remarkable duality, which has been observed in the nonlinear regime for the electromagnetic response of quantum Hall systems.
\end{abstract}

DOI: 10.1103/PhysRevB.65.155323

PACS number(s): 73.43.Cd, 11.10.Ef

\section{INTRODUCTION}

There is now a good understanding of the physical processes underlying the quantum Hall effect, at least at the Hall plateaux where the Laughlin wave functions and the Jain hierarchy give accurate and insightful descriptions of the observed phenomena. However, the transition between plateaux, as external quantities (e.g., magnetic field) are varied, is less well understood theoretically, despite there now being a wealth of experimental data on these crossovers.

A milestone in this understanding was the observation that many features of the critical points of these transitions were "superuniversal"1 (see, however, Ref. 2), and the subsequent interpretation of this in terms of an underlying symmetry. 5,6 Unfortunately, the resulting understanding of this symmetry in terms of microscopic physics ${ }^{5}$ - the law of corresponding states-suffers from two related drawbacks. First, it relies on what are ultimately uncontrolled (mean-field) approximations when making contact with quantum Hall observables. Second, the derivation suggests that the domain of validity of the symmetries is more restricted than what appears in experiments, being apparently restricted to the domain of linear response and to the immediate vicinity of the critical points of the transitions between plateaux.

In an earlier paper ${ }^{7}$ we argued that many of the consequences of Kivelson, Lee, and Zhang's analysis could be understood away from the critical points and without making the mean-field approximation. In a nutshell, this was done by deriving them from an effective low-energy theory consisting of electromagnetically interacting quasiparticles or vortices. In two space dimensions the interactions of particles and vortices are described by similar Lagrangians, and it is the symmetries that follow from this similarity, which underlie the success of the law of corresponding states. These successes may, therefore, be seen to follow as predictions for any system for which the low-energy electromagnetic response can be shown to be well described by the effective theory we propose.

Here we extend the discussion of Ref. 7 to applications that are beyond the approximation of linear response. In particular, we shall derive duality relations for the electromagnetic response, which apply even in the nonlinear regime. In so doing we provide the theoretical explanation of the remarkable symmetry under interchange of current and (longitudinal) voltage, which has been measured near the critical point of the transition between Laughlin plateaux and the Hall insulator. ${ }^{8}$

We begin, in the following section, by reviewing the main features of the description of the low-energy electromagnetic response given in Ref. 7. This is followed, in Sec. III, by the extension of this description beyond the regime of linear response.

\section{THE EFFECTIVE THEORY}

Our starting point is the recognition that the energies associated with electromagnetic response experiments are much lower than the typical microscopic electronic energies. For instance, the activation energy as measured by the temperature dependence of the Ohmic resistivity is of order $E_{r}$ $\sim 0.1 \mathrm{~K} \sim 10 \mu \mathrm{eV}$, as compared to the underlying Coulomb and cyclotron energies which are of order $E_{c} \sim 100 \mathrm{~K}$ $\sim 10 \mathrm{meV}$.

All experiments are, in principle, described by a microscopic Hamiltonian describing the conduction electrons and their interactions, but accurate calculations with this Hamiltonian are difficult to perform. Although it is not strictly necessary for our later discussion, it is useful to imagine this effective theory to be written in the manner of Kivelson, Lee, and Zhang, as a system of interacting bosons described by a field $\Phi$ coupled to a statistics field $a_{\mu}$ with an odd statistics parameter $\theta=(2 n+1) \pi$. (As is well known, such a system is exactly equivalent in two dimensions to interacting fermions. ${ }^{9}$ )

Due to the large hierarchy, $E_{r} \ll E_{c}$, one can imagine integrating out the largely irrelevant high-energy dynamics to derive an effective Lagrangian with which to describe the low-energy experiments. Although the direct, first-principles calculation of the low-energy effective theory is usually as difficult as solving the full microscopic model, progress may be made inasmuch as the low-energy degrees of freedom are more weakly interacting than those at higher energies. In this case it can be possible to extract precise predictions within controllable approximations, even when the same cannot be done with the full underlying microphysical system. ${ }^{10-12}$ When this is possible, direct appeal to the microscopic theory is only required to establish the validity of the assumed low-energy degrees of freedom, and it need not play a crucial quantitative role in the comparison with experiment.

Our key assumption here, and in Ref. 7, is that these 
techniques may be applied to quantum Hall systems, where we will assume that the low-energy dynamics can be described by a system of weakly interacting charged quasiparticles or vortices. The quasiparticles need not be electrons, which could well be strongly interacting in the microscopic theory, but are taken to be some effective description of the low-energy physics. For instance, motivated by the composite fermion picture, ${ }^{13}$ we take the quasiparticles to be fermions when describing the Laughlin plateaux [for which $\sigma_{x y}$ $=1 /(2 n+1)$ in our units, with $\left.e^{2} / h=1\right]$. On the other hand vortices will be assumed instead to govern the low-energy response of the Hall insulator.

More concretely, for those phases described by quasiparticles, following Ref. 7, we use the following effective Lagrangian, describing the low-energy/long-wavelength interaction of a collection of bosonic charged quasiparticles, coupled to electromagnetic, $A_{\mu}$, and statistical gauge fields, $a_{\mu}$, with statistical angle $\theta$ :

$$
\mathcal{L}_{\theta}(\xi, a, A)=-\frac{\pi}{2 \theta} \epsilon^{\mu \lambda \nu} a_{\mu} \partial_{\lambda} a_{\nu}+\mathcal{L}_{p}(\xi, a+A)
$$

Here $\mathcal{L}_{p}(\xi, a+A)$ is the Lagrangian for the quasiparticles, where $\xi_{k}$ is the position of the $k$ th particle,

$$
\mathcal{L}_{p}=\sum_{k}\left[\frac{m}{2} \dot{\xi}_{k}^{\mu} \dot{\xi}_{k \mu}-q \dot{\xi}_{k}^{\mu}(a+A)_{\mu}-V(\xi)\right] \delta\left(x-\xi_{k}(t)\right),
$$

where $m$ is the quasiparticle mass, $q$ is the charge, and $V(\xi)$ is a potential representing other quasiparticle interactions with their environment. Equation (2) represents the first few terms of a derivative expansion of the low-energy quasiparticle Lagrangian.

For the present applications it is important to keep in mind that the electromagnetic field, $A_{\mu}$, which appears in this effective theory is itself a low-energy effective field. It does not, in particular, include the large background magnetic field $B$ whose presence the quantum Hall effect requires. (Indeed, it cannot include such a large field, since motion within this field would involve energies of order $\omega_{c}$ $=e B / m$, which have been integrated out to obtain the lowenergy theory.) $A_{\mu}$ instead represents all of the weaker fields in the low-energy part of the problem, including, in particular, those fields that are applied in order to describe the system's electromagnetic response. The dependence of lowenergy quantities on the background field $B$ is implicit in all of the parameters of the effective theory, such as in the total number of particles or vortices, the particle/vortex masses and couplings, etc.

For those phases whose low-energy behavior is described by vortices, we instead use the general vortex action, which for our purposes has a very convenient representation in terms of the vortex positions $y_{k}$ and a new gauge potential $b_{\mu}$, which is a dual representation of the scalar field, which mediates the long-range interactions amongst vortices, ${ }^{9}$

$$
\begin{aligned}
\widetilde{\mathcal{L}}_{\theta}(y, a, b, A)= & -\frac{\pi}{2 \theta} \epsilon^{\mu \lambda v} a_{\mu} \partial_{\lambda} a_{\nu}-\epsilon^{\mu \lambda \nu} b_{\mu} \partial_{\lambda}\left(a_{\nu}+A_{\nu}\right) \\
& +\mathcal{L}_{v}(y, b) .
\end{aligned}
$$

Here $\mathcal{L}_{v}(y, b)$ is the Lagrangian for the vortex motion,

$$
\mathcal{L}_{v}=\sum_{\widetilde{k}}\left[\frac{\tilde{m}}{2} \dot{y}_{\tilde{k}}^{\mu} \dot{y}_{\tilde{k} \mu}-\tilde{q} \dot{y}_{\tilde{k}}^{\mu} b_{\mu}-\widetilde{V}(y)\right] \delta\left(x-y_{\tilde{k}}(t)\right),
$$

with $\tilde{m}$ the vortex mass, $\tilde{q}$ the vortex charge (governing its coupling to the field $b_{\mu}$ ), and $\widetilde{V}(y)$ represents possible vortex interaction terms.

The central property, which we now assume of the quasiparticle and vortex effective Lagrangians and which underlies our subsequent conclusions, is that $\mathcal{L}_{p}(\xi, a)$ and $\mathcal{L}_{v}(y, b)$ have the same functional form when considered as functionals of their respective arguments, $(\xi, a)$ or $(y, b)$. A sufficient condition for this to be true-at least at the lowest orders of the derivative expansion, which suffice in the low-energy limit - is that the interactions with the environment, $V(\xi)$ and $V(y)$, be negligible. (Although sufficient, this condition might not be absolutely necessary.)

It remains an intractable problem to solve even these effective theories in any generality. However, if $\mathcal{L}_{p}(\xi, a)$ and $\mathcal{L}_{v}(y, b)$ do have the same functional form it is possible to relate the electromagnetic response for a system of vortices to the response for a similar system of quasiparticles. It is this relationship that we now derive, without making the assumption of linear response in the fields $A_{\mu}$.

\section{THE EXPERIMENTS}

Before diving into the implications of particle-vortex similarity, it is worth describing the evidence for particlevortex duality beyond linear response. Besides being an interesting topic in its own right, a description of these experimental results provides a sharper statement of what it is that must be derived in the subsequent sections.

The central nonlinear result is summarized by Fig. 1, which is reproduced from Ref. 8. Each curve in this figure represents a trace of the longitudinal (Ohmic) current $I_{x}$ plotted against the longitudinal voltage $V_{x}$. The different curves are taken for different values of the applied magnetic field, as the magnetic field is varied across the transition between the $\sigma_{x y}=1$ quantum Hall plateau and the Hall insulator. The solid lines are all traces taken on one side of this transition, while the dotted lines are taken on the other side.

What is remarkable about this figure is its symmetry about reflection through the diagonal axis $I_{x}=V_{x}$. Traces taken on one side of the transition are very accurately the reflections of those taken on the other side of the transition. What is not shown in this figure, but is demonstrated in Ref. 8, is that the filling factors $\nu$ corresponding to the mirror-image traces are spaced an equal distance away, $\Delta \nu$ $=\left|\nu-\nu_{c}\right|$, from the critical filling factor.

The linear-response regime in these plots corresponds to the straight segments near the origin, and within this regime the reflected current/voltage curves have slopes that corre- 


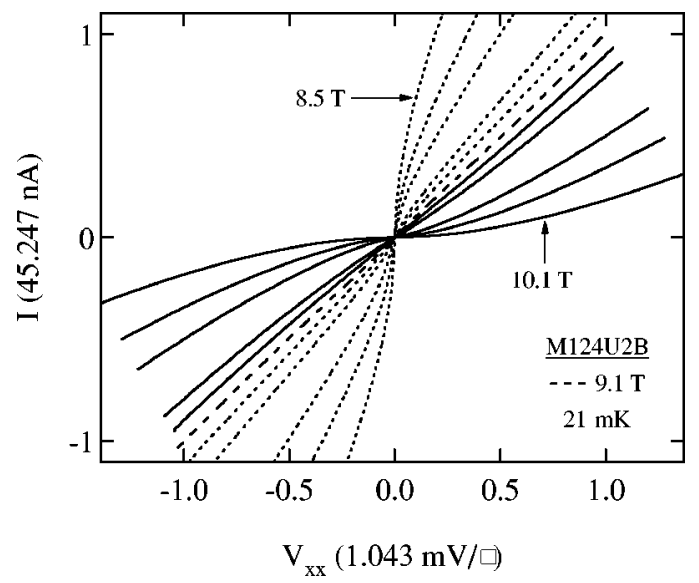

FIG. 1. Longitudinal current vs voltage in quantum Hall systems, taken for several magnetic fields on either side of the critical point in the $\sigma_{x y}=1$ to Hall insulator transition (reproduced from Ref. 8). The solid lines correspond to one side of the transition and the dotted lines to the other side. Notice how the solid lines are reflections of the dotted lines about the diagonal line $I=V$, as would be expected for particle-vortex interchange.

spond to resistivities that are related by $\tilde{\rho}_{x x}=\rho_{c}^{2} / \rho_{x x}$, where $\rho_{c}=\rho_{x x}\left(\nu_{c}\right)=1$ is the universal value of the critical Ohmic resistivity for this transition.

While $\rho_{x x}$ is varying as just described, what does $\rho_{x y}$ do? The experiments show that $\rho_{x y}$ is a constant and so does not vary at all through the transition. ${ }^{8}$

In Ref. 7 it was shown that if the particle and vortex effective Lagrangians have the same functional form as assumed above and if the quasiparticles are fermions (or related to fermions in a precise way), then this imposes a remarkable constraint on the trajectory followed by a system in the conductivity plane as external parameters (like $B$ ) are varied. The constraint is that the trajectory must commute with a discrete group $\Gamma_{0}(2)$, which may be defined by the transformation $\sigma \rightarrow(a \sigma+b) /(2 c \sigma+d)$, where the integers $a, b, c$, and $d$ satisfy $a d-2 b c=1$. Here $\sigma$ denotes the convenient complex quantity $\sigma=\sigma_{x y}+i \sigma_{x x}$.

This symmetry provides an excellent description of Fig. 1 specialized to the linear-response regime. ${ }^{14}$ First, the symmetry predicts the critical resistivity in transitions between plateaux to be universal, and to be given by $\rho_{c}=1$ for transitions to the Hall insulator from the Laughlin sequence. Second, it also implies for these transitions that $\rho_{x y}$ must be constant throughout the transition (called the "semicircle law" because these trajectories are semicircles when drawn in the $\sigma$ plane). Finally, there is a symmetry generator that maps each semicircle trajectory onto itself, but with end points reversed, which corresponds precisely to particlevortex interchange. As is shown in Ref. 14, this symmetry element is precisely equivalent to the observed symmetry.

For instance, for transitions between the $\nu=1$ plateau and the Hall Insulator (which are along the semicircle centered at $\sigma=\frac{1}{2}$, linking $\sigma=1$ and $\sigma=0$ ) this symmetry acts in the following way:

$$
\tilde{\sigma}=\frac{\sigma-1}{2 \sigma-1}
$$

Once restricted to the semicircular trajectories in the $\sigma$ plane-which correspond to curves having constant $\rho_{x y}$-Eq. (5) is precisely equivalent to the statement $\tilde{\rho}_{x x}$ $=1 / \rho_{x x}$. The analogous symmetry for other transitions is obtained from this by acting with the group element, which takes $(0,1)$ to the desired end points $\left(p_{1} / q_{1}, p_{2} / q_{2}\right)$. For example, for the $1 / 3 \rightarrow 0$ transition this gives the symmetry generator $\tilde{\sigma}=(3 \sigma-1) /(10 \sigma-3)$, which again corresponds to $\tilde{\rho}_{x x}=1 / \rho_{x x}$.

To describe the data beyond linear response we must show that the curves $I_{x}\left(V_{x}\right)$ get mapped into their inverses, $\widetilde{V}_{x}\left(\widetilde{I}_{x}\right)$, by the action of interchanging particles and vortices. This is most easily demonstrated by proving the equivalent statement for the tangents to these curves, which is

$$
\tilde{\rho}_{x x}\left(\widetilde{I}_{x}\right)=\frac{d \widetilde{V}_{x}}{d \widetilde{I}_{x}}=\frac{d I_{x}}{d V_{x}}=\frac{1}{\rho_{x x}\left(I_{x}\right)},
$$

when evaluated along a trajectory for which $\rho_{x y}$ is constant. Notice that the only difference between Eq. (6) and the corresponding result in linear response is the dependence on $I_{x}$ and $\widetilde{I}_{x}$, which is allowed in Eq. (6) but not in linear response.

\section{PARTICLE-VORTEX DUALITY IN THE NONLINEAR REGIME}

We now show how Eq. (6) follows from the similarity of the quasiparticle and vortex effective Lagrangians. We do so in two steps. The first step, already given in Ref. 7, is to derive an expression relating the quasiparticle nonlinear electromagnetic response for $\theta=\pi$ to the vortex nonlinear response for $\theta=-\pi$. (Both $\theta=\pi$ and $\theta=-\pi$, or any other odd multiple of $\pi$, are appropriate for transitions from the Laughlin plateaux since the quasiparticles in this case are fermions, such as in the composite fermion picture.) The second step, proposed in this paper, is to derive an exact expression for how the electromagnetic response for vortices varies when $\theta$ is changed from $\pi$ to $-\pi$.

\section{A. Step I}

We start with a system of fermionic quasiparticles described using $\theta=-\pi$ in Eq. (1). This gives

$$
\begin{aligned}
\mathcal{L}_{\theta=-\pi}(\xi, a, A)= & +\frac{1}{2} \epsilon^{\mu \nu \lambda} a_{\mu} \partial_{\nu} a_{\lambda}+\mathcal{L}_{\text {kin }}(\xi) \\
& +j^{\mu}(\xi)(a+A)_{\mu}-V(\xi) .
\end{aligned}
$$

On the other hand, the vortex system with $\theta=\pi$ in Eq. (3) gives

$$
\begin{aligned}
\widetilde{\mathcal{L}}_{\theta=\pi}(y, a, b, A)= & -\frac{1}{2} \epsilon^{\mu \nu \lambda} a_{\mu} \partial_{\nu} a_{\lambda}-\epsilon^{\mu \nu \lambda} b_{\mu} \partial_{\nu}(a+A)_{\lambda} \\
& +\widetilde{\mathcal{L}}_{\text {kin }}(y)+\widetilde{j}^{\mu}(y) b_{\mu}-\widetilde{V}(y) .
\end{aligned}
$$


These are used to generate the electromagnetic response functions $\Gamma_{\theta}[A]$ and $\widetilde{\Gamma}_{\theta}[A]$ by evaluating the following path integrals:

$$
\begin{aligned}
e^{(i / \hbar) \Gamma_{\theta}[A]}= & \int\left[d a_{\mu}(x)\right] \prod_{k}\left[d \xi_{k}^{\mu}(t)\right] \\
& \times \exp \left[\frac{i}{\hbar} \int d^{3} x \mathcal{L}_{\theta}(\xi, a, A)\right]
\end{aligned}
$$

and

$$
\begin{aligned}
e^{(i / \hbar) \widetilde{\Gamma}_{\theta}[A]}= & \int\left[d a_{\mu}(x)\right]\left[d b_{\mu}(x)\right] \prod_{\widetilde{k}}\left[d y_{\tilde{k}}^{\mu}(t)\right] \\
& \times \exp \left[\frac{i}{\hbar} \int d^{3} x \widetilde{\mathcal{L}}_{\theta}(y, a, b, A)\right] .
\end{aligned}
$$

Of course neither $\Gamma_{\theta=-\pi}[A]$ nor $\Gamma_{\theta=\pi}[A]$ can be calculated exactly, but the followings argument implies a relation between them that must always be true in the long-wavelength limit so long as the quasiparticles and vortices interactions at low energy are similar (or negligible).

Our goal for these two systems is to use the similarity of the Lagrangians to relate the results of performing the path integrations. To see this relation first shift $b_{\mu} \rightarrow b_{\mu}+A_{\mu}$ in Eq. (10), and then perform the Gaussian integral over $a_{\mu}$. The resulting effective Lagrangian is

$$
\begin{aligned}
\widetilde{\mathcal{L}}_{\theta=\pi}(y, b, A)= & +\frac{1}{2} \epsilon^{\mu \nu \lambda} b_{\mu} \partial_{\nu} b_{\lambda}-\frac{1}{2} \epsilon^{\mu \nu \lambda} A_{\mu} \partial_{\nu} A_{\lambda}+\widetilde{\mathcal{L}}_{\mathrm{kin}}(y) \\
& +\widetilde{j}^{\mu}(y)(b+A)_{\mu}-\widetilde{V}(y) \\
= & \widetilde{\mathcal{L}}_{\theta=\pi}^{\prime}(y, b, A)-\frac{1}{2} \epsilon^{\mu \nu \lambda} A_{\mu} \partial_{\nu} A_{\lambda}
\end{aligned}
$$

where the second step defines $\widetilde{\mathcal{L}}_{\theta}^{\prime}$.

The main point is that $\widetilde{\mathcal{L}}_{\theta=\pi}^{\prime}$ has the same form as does $\mathcal{L}_{\theta=-\pi}$, to the extent that both $\widetilde{\mathcal{L}}_{\text {kin }}(y)$ and $\mathcal{L}_{\text {kin }}(\xi)$ and $\tilde{j}^{\mu}(y)$ and $j^{\mu}(\xi)$ have the same functional form. This ensures that $\widetilde{\Gamma}_{\theta=\pi}[A]$ is related to $\Gamma_{\theta=-\pi}[A]$ by

$$
\widetilde{\Gamma}_{\theta=\pi}[A]=\Gamma_{\theta=-\pi}[A]-\frac{1}{2} \int d^{3} x \epsilon^{\mu \nu \lambda} A_{\mu} \partial_{\nu} A_{\lambda},
$$

even though we cannot calculate either explicitly. Notice that Eq. (12) goes beyond linear response- there is no need to assume that $\Gamma_{\theta}[A]$ or $\widetilde{\Gamma}_{\theta}[A]$ is quadratic in $A$.

The relation between the nonlinear conductivities [such as $\left.\sigma_{x x}\left(V_{x}\right)\right]$ of the particle and vortex systems is now obtained by differentiating the response function to obtain the polarization tensor

$$
\Pi_{\theta}^{\mu \nu}=-\frac{\delta^{2} \Gamma_{\theta}[A]}{\delta A_{\mu} \delta A_{\nu}} .
$$

For a conductor the Fourier-transformed quantity $\Pi_{\theta}^{\mu \nu}(\omega, \mathbf{p})$ vanishes linearly at $\omega=0$ and the conductivity is defined by

$$
\sigma_{\alpha \beta}^{\theta}(A)=-i \lim _{\omega \rightarrow 0}\left[\Pi_{\theta}^{\alpha \beta}(\omega, \mathbf{0}) / \omega\right],
$$

where $\alpha, \beta=x, y$. Notice that both of these definitions also apply in the nonlinear regime, so long as Eq. (13) is not evaluated at zero field: $A_{\mu}=0$.

For the dual system we see that Eq. (12) implies

$$
\widetilde{\Pi}_{\theta=\pi}^{\mu \nu}(p)=\Pi_{\theta=-\pi}^{\mu \nu}(p)+i \epsilon^{\mu \lambda \nu} p_{\lambda},
$$

and so the nonlinear complex conductivities are related by

$$
\tilde{\sigma}_{\theta=\pi}=\sigma_{\theta=-\pi}+1 .
$$

This is essentially the Landau-level addition transformation of Kivelson, Lee, and Zhang, ${ }^{5}$ extended here to the nonlinear regime. The nonlinear argument given here was first presented in Ref. 7.

\section{B. Step II: An aside}

We next examine the effect on the electromagnetic response of a $2 \pi$ shift of $\theta$ without interchanging particles with vortices (or varying other external parameters). Once this is known, it may be combined with Eq. (16) to give the effect of particle-vortex interchange without simultaneously shifting $\theta$. What is different about this discussion is its derivation beyond the regime of purely linear response.

Before deriving the result of a $2 \pi$ shift in $\theta$ we shall pause to consider what it means. Indeed, one might reasonably expect that all physical quantities - and, in particular, the conductivities-should be strictly periodic with respect to $\theta \rightarrow \theta+2 \pi$. To see why this need not be so in the effective theory, we first review why it is true for the microscopic theory.

Within first-quantized theory the action describing the coupling of the statistics field, $a_{\mu}$, to particles is strictly quadratic. The path integral over $a_{\mu}$, is therefore, Gaussian and is equivalent (up to an overall field-independent normalization) to evaluating the action at its stationary point $a_{\mu}$ $=a_{\mu}^{c}$. Since this configuration has a vanishing field strength, $f_{\mu \nu}^{c}=0$ (away from the position of any of the particles to which it couples) it is locally pure gauge. The integral $\oint a_{\mu}^{c} d x^{\mu} \neq 0$ about any curve that encloses particle sources, however; so there is some physics in $a_{\mu}^{c}$ and this physics encodes the statistics phases that accrue whenever two particles exchange positions. ${ }^{15}$

If the particles involved all have hard cores and so can never interpenetrate one another, then the particle positions may be excised and the physics of the statistics field comes purely from topology. In this case the above picture gives the whole story, $a_{\mu}$ purely encodes particle statistics, and all physical quantities are strictly periodic in $\theta$. This is the situation for the microscopic electrons, as described in the quantum Hall context in Ref. 9.

The picture changes if the source for $a_{\mu}$ is distributed continuously. Consider, for example, a uniform distribution of "charge," which gives rise to a uniform distribution of statistical-field magnetic flux. In this case the statistics field is not pure gauge since $f_{\mu \nu}^{c} \neq 0$, and its magnetic part is proportional to the source density. Consequently the physics can depend on the local values of $a_{\mu}$. Since $a_{\mu}$ couples to $A_{\mu}$ 
only through the combination $(a+A)_{\mu}$, particles see this magnetic statistics field as an addition to the real magnetic field $B$.

For continously distributed source distributions, since $a_{\mu}$ encodes more than statistics phases, there is no need for physical quantities to be periodic under $\theta \rightarrow \theta+2 \pi$. Such aperiodicity might be expected to occur in phases of the theory for which quasiparticles or vortices have condensed to form a nontrivial ground state.

For quantum Hall systems we are led to a picture very much like the one that arises in Ref. 5. For the microscopic electrons the physics is strictly periodic under $2 \pi$ shifts of the statistics angle $\theta$. However, the system has a great many phases, and the effective theory built over the ground state of any particular phase need not be invariant under these shifts of the statistics angle. The periodicity of the full theory is seen once all of these phases are viewed together, since changes to $\theta$ take one phase into another. The change of phase can be understood qualitatively because changes in $\theta$ cause changes to $a_{\mu}^{c}$, which may be compensated by changes in $A_{\mu}$, and, in particular, in the applied magnetic field. But changing the applied magnetic field is one of the methods used to move between different phases in the lab.

Thus, the underlying invariance with respect to $\theta \rightarrow \theta$ $+2 \pi$ emerges in the effective theory as a relation between the properties of different phases of the system, with the physics of any individual phase not being simply periodic. We may legitimately ask what the effect of such a shift is on the electromagnetic response of the system.

\section{Step II: The calculation}

We now proceed with the calculation of the effects of a $2 \pi$ shift of $\theta$ on the nonlinear response function $\Gamma_{\theta}[A]$. To this end consider the generating function $W_{\theta}[J]$ for the electromagnetic correlation functions:

$$
\exp \left(\frac{i}{\hbar} W_{\theta}[J]\right)=\int[d A] \exp \left(\frac{i}{\hbar} \Gamma_{\theta}[A]+\frac{i}{\hbar} \int d^{3} x A_{\mu} J^{\mu}\right),
$$

where $\Gamma_{\theta}[A]$ is defined by Eqs. (1) and (9). To perform the $a$ integral, shift $A_{\mu} \rightarrow B_{\mu}:=A_{\mu}+a_{\mu}$, so that the statistics field $a_{\mu}$ only appears in the Chern-Simons term and through the current coupling $\int d^{3} x\left(B_{\mu}-a_{\mu}\right) J^{\mu}$. The $a_{\mu}$ integral may then be explicitly performed, since it is Gaussian. The result is the following (neglecting as usual overall factors):

$$
\begin{aligned}
\exp \left(\frac{i}{\hbar} W_{\theta}[J]\right)= & \int[d B] \exp \left\{\frac{i}{\hbar} S[B]+\frac{i}{\hbar} \int d^{3} x B_{\mu} J^{\mu}\right. \\
& \left.-\frac{i}{\hbar}\left(\frac{\theta}{2 \pi}\right) \iint d^{3} x d^{3} x^{\prime} \epsilon^{\mu \nu \lambda} J_{\mu}\left(\frac{1}{\partial^{2}}\right) \partial_{\nu} J_{\lambda}\right\},
\end{aligned}
$$

where $e^{(i / \hbar) S[B]}=\int \Pi_{k}\left[d \xi_{k}\right] \exp \left[i / \hbar \int d^{3} x \mathcal{L}_{p}(\xi, B)\right]$.

This makes the $\theta$ dependence of $W_{\theta}[J]$ explicit, and shows that

$$
W_{\theta}[J]=W_{0}[J]-\left(\frac{\theta}{2 \pi}\right) \iint d^{3} x d^{3} x^{\prime} \epsilon^{\mu \nu \lambda} J_{\mu}\left(\frac{1}{\partial^{2}}\right) \partial_{\nu} J_{\lambda}^{\prime} .
$$

To make contact with the polarization tensor $\Pi_{\theta}^{\mu \nu}$ we must relate $W_{\theta}[J]$ to $\Gamma_{\theta}[A]$. To within a very good approximation they are Legendre transforms of one another. That is, defining the Legendre transform $L_{\theta}[A]$ of $W_{\theta}(J)$ by

$$
L_{\theta}[A]=W[J]-\int d^{3} x A_{\mu} J^{\mu}
$$

with $A_{\mu}=\delta W_{\theta} / \delta J^{\mu}$, standard field-theoretic arguments imply that $L_{\theta}$ is related to $\Gamma_{\theta}$ in the following way:

$e^{(i / \hbar) L_{\theta}[A]}=\int\left[d A^{\prime}\right] \exp \left\{\frac{i}{\hbar}\left[\Gamma_{\theta}\left[A^{\prime}+A\right]+\int A_{\mu}^{\prime} J^{\mu}[A] d x\right]\right\}$,

where $J^{\mu}=-\delta L_{\theta} / \delta A_{\mu}$. It follows that $\Gamma_{\theta}[A]$ and $L_{\theta}$ are equal to one another if the $A_{\mu}^{\prime}$ integral is performed semiclassically. Since the low-energy applied electromagnetic fields used in linear response, $A_{\mu}$, are very well described semiclassically, we can equate $L_{\theta}$ and $\Gamma_{\theta}$ to an equally good approximation.

It then follows that the derivative $W_{\mu \nu}^{\theta}=\delta^{2} W_{\theta} / \delta J^{\mu} \delta J^{\nu}$ is related to $\Pi_{\theta}^{\mu \nu}$ by $\Pi_{\theta}^{\mu \nu} W_{\nu \lambda}^{\theta}=\Lambda_{\lambda}^{\mu}$, where $\Lambda_{\mu \nu}=\eta_{\mu \nu}$ $-p_{\mu} p_{\nu} / p^{2}$. We use here (for convenience of notation only) a relativistic notation with $\eta_{\mu \nu}=\operatorname{diag}(-1,1,1,1){ }^{7}$ For brevity we write this relation as $W_{\mu \nu}^{\theta}=\left(\Pi_{\theta}^{\mu \nu}\right)^{-1}$.

Combining the above results, in momentum space we have

$$
\left(\Pi_{\theta}^{\mu \nu}\right)^{-1} \approx\left(\Pi_{0}^{\mu \nu}\right)^{-1}+\left(\frac{\theta}{\pi}\right) \frac{\hbar}{\sqrt{p^{2}}} \mathcal{J}^{\mu \nu},
$$

where $\mathcal{J}_{\mu \nu}=i \epsilon_{\mu \lambda \nu} p^{\lambda} / \sqrt{p^{2}}$.

For $\theta=2 \pi$ this reproduces the results of Ref. 7 for the flux attachment transformation for the conductivities,

$$
-\frac{1}{\tilde{\sigma}}=-\frac{1}{\sigma}+2,
$$

where $\tilde{\sigma}$ is obtained from $\Pi_{\theta}^{\mu \nu}$ as in Eq. (14). This is the flux attachment transformation of Ref. 5, extended again to the nonlinear regime. The only difference between Eq. (23) and the linear-regime results of Ref. 7 is that here $\sigma$ can be a function of the external electromagnetic effective field.

\section{Particle-vortex interchange}

Our goal is to derive Eq. (6) as the effect of particlevortex interchange (at fixed $\theta$ ), and so we must combine the results of Eqs. (16) and (23).

The simplest way to do so is to recognize the group $\Gamma_{0}(2)$, which is obtained through repeated applications of Eqs. (16) and (23). ${ }^{5,6,16}$ A familiar form for this group structure is most easily seen by writing it in terms of the two operations 


$$
T: \sigma \rightarrow \sigma+1, \quad S: \sigma \rightarrow-\frac{1}{\sigma},
$$

which satisfy $(S T)^{3}=1$. In terms of these operations the group of interest $\left[\Gamma_{0}(2)\right]$ is generated by

$$
T: \sigma \rightarrow \sigma+1, \quad S T^{2} S: \sigma \rightarrow \frac{\sigma}{1-2 \sigma}
$$

The operation $S$ is only introduced here for convenience. It is not a symmetry of the quantum Hall effect, since it cannot be obtained by repeated applications of the basic transformations (16) and (23). ( $S$ represents interchange of the conductivity and the resistivity. Although it is not a symmetry of the quantum Hall effect, for which the charge carriers are fermions, it should be a symmetry for twodimensional systems in which the charge carriers are bosonic. $^{7}$ )

Combining the two operations $T$ and $S T^{2} S$ we see that the effect of interchanging fermionic quasiparticles and vortices is given by

$$
T S T^{2} S(\sigma)=\frac{\sigma-1}{2 \sigma-1},
$$

which is precisely Eq. (5), although now generalized to the nonlinear regime by including field-dependent $\sigma$.

As was discussed earlier, this provides a successful description of the nonlinear duality of the transition between the $\sigma_{x y}=1$ plateau and the Hall insulator. Since the group structure is the same as in the linear-response regime, we may now repeat the linear-response $\operatorname{arguments}^{14}$ to immediately understand the analogous result for particle-vortex interchange in the transitions to the Hall insulator from the Laughlin sequence, $\sigma_{x y}=1 /(2 n+1)$, despite the fact that the quasiparticles in this instance enjoy fractional statistics, and so are no longer fermions.

To understand the $\nu=\frac{1}{3} \rightarrow 0$ transition in the form presented here, we obtain it as a symmetry transformation of the $\nu=1 \rightarrow 0$ transition just described. The modular symmetry (25) dictates that the $1 \rightarrow 0$ transition follows a semicircle in the complex $\sigma$ plane, with the critical point at $\sigma_{c}=(1$ $+i) / 2,{ }^{14}$ and we have found the quasiparticle-vortex duality to be implemented by the transformation, Eq. (26).

This transformation interchanges the end points $0 \leftrightarrow 1$ and leaves the critical point, $\sigma_{c}$, fixed. To study other transitions, such as the $\nu=\frac{1}{3} \rightarrow 0$ transition examined experimentally, we must find the group element that maps this basic semicircle of radius $\frac{1}{2}$, arching between $\sigma=1$ and $\sigma=0$, onto the semicircle of radius $\frac{1}{6}$, arching between $\sigma=\frac{1}{3}$ and $\sigma=0$. Once found, this group element can be used to transform Eq. (5) into the transformation appropriate for particle-vortex interchange in the $\frac{1}{3} \rightarrow 0$ transition. The result of this exercise is the transformation $\sigma \rightarrow(3 \sigma-1) /(10 \sigma-3)$. As is easily checked, this interchanges $\sigma=\frac{1}{3}$ and $\sigma=0$ and maps the critical point $\sigma_{c}=(3+i) / 10$ to itself.

Once mapped to the resistivity plane, the transition is again along the line with constant $\rho_{x y}=3$, along which the particle-vortex interchange becomes $\rho_{x x} \rightarrow 1 / \rho_{x x}$. This shows that the experimental observations of Ref. 8 are a consequence of the particle-vortex interchange, even deep within the nonlinear regime. Just as for linear response, ${ }^{7}$ this effective field theory analysis sheds light on why the duality is experimentally successful so far from the critical points, to which the analysis of Ref. 5 was believed to be restricted.

In conclusion we have shown that the law of corresponding states is applicable in the nonlinear regime-well outside of the linear regime of its original derivation. In particular the pseudoparticle-vortex duality, which was invoked to explain the experiments in Ref. 8, can be extended into the nonlinear regime, as is necessary to explain these experimental observations.

Our arguments assume the long-wavelength, low-energy limit and are applicable to any system in which the interactions between the pseudoparticles are weak, as are the interactions between the vortices, so that there is a symmetry under interchange of pseudoparticles and vortices. More generally one could allow stronger interactions between the pseudoparticles, provided the interaction potential between vortices is of the same form, but this might be harder to realize in practice.

For fermionic pseudoparticles the resulting symmetry group, is $\Gamma_{0}(2)$ and this group therefore, seems to be the one relevant to the quantum Hall effect (other candidates have also been considered, ${ }^{17}$ which may be the relevant symmetries when electron spins are not well separated by Zeeman splitting. ${ }^{18}$ ) It was argued in Ref. 7 that a different group is relevant when the pseudoparticles are bosonic-the group given by the matrix $\gamma=\left(\begin{array}{l}a b \\ c d\end{array}\right)$ with $a, d$ both even and $b, c$ both odd or vice versa. This group is often denoted by $\Gamma_{\theta}(2)$ in the mathematical literature and is generated by $S$ and $T^{2}$. The arguments presented here are, of course, just as applicable to such bosonic systems.

\section{ACKNOWLEDGMENTS}

We thank A. MacDonald and J. Maldacena for helpful discussions. C.P.B. is grateful to Aspen Centre for Physics where part of this work was carried out. Our research has been assisted by financial support from N.S.E.R.C. (Canada), F.C.A.R. (Québec), the Ambrose Monell Foundation, and Enterprise Ireland, Basic Research, Grant No. SC/1998/739.

\footnotetext{
*Email address: cliff@physics.mcgill.ca

†Email address: bdolan@thphys.may.ie

${ }^{1}$ H.P. Wei, D.C. Tsui, M.A. Paalanen, and A.M.M. Pruisken, Phys.

Rev. Lett. 61, 1294 (1988); L. Engel et al., Surf. Sci. 229, 13 (1990).

${ }^{2}$ The experimental situation more recently has become more
}

murky, with new evidence for $^{3}$ and against ${ }^{4}$ scaling behavior. We predict scaling and universality to occur in any system to which the particle-vortex duality we propose applies.

${ }^{3}$ P.T. Coleridge, Phys. Rev. B 60, 4493 (1999); P.T. Coleridge, Solid State Commun. 112, 241 (1999).

${ }^{4}$ D. Shahar, M. Hilke, C.C. Li, D.C. Tsui, S.L. Sondhi, and M. 
Razeghi, Solid State Commun. 107, 19 (1998).

${ }^{5}$ D.-H. Lee, S. Kivelson, and S.-C. Zhang, Phys. Rev. Lett. 68, 2386 (1992); S. Kivelson, D.-H. Lee, and S.-C. Zhang, Phys. Rev. B 46, 2223 (1992).

${ }^{6}$ C.A. Lütken and G.G. Ross, Phys. Rev. B 45, 11837 (1992); 48, 2500 (1993).

${ }^{7}$ C.P. Burgess and B.P. Dolan, Phys. Rev. B 63, 155309 (2001).

${ }^{8}$ D. Shahar, D.C. Tsui, and M. Shayegan, Science 274, 589 (1996).

${ }^{9}$ For a review with references see, S.-C. Zhang, Int. J. Mod. Phys. B 6, 25 (1992).

${ }^{10}$ S. Weinberg, Prog. Theor. Phys. Suppl. 86, 43 (1986).

${ }^{11}$ J. Polchinski, hep-th/9210046 (unpublished).

${ }^{12}$ R. Shankar, Rev. Mod. Phys. 66, 129 (1994).
${ }^{13}$ For a review of the composite fermion picture see, e.g., Composite Fermions: A Unified View of the Quantum Hall Regime, edited by O. Heinonen (World Scientific, Singapore, 1998).

${ }^{14}$ C.P. Burgess, Rim Dib, and B.P. Dolan, Phys. Rev. B 62, 15359 (2000).

${ }^{15}$ D. Arovas, J.R. Schrieffer, and F. Wilczek, Phys. Rev. Lett. 53, 722 (1984).

${ }^{16}$ C.A. Lütken, Nucl. Phys. B 396, 670 (1993).

${ }^{17}$ Y. Georgelin and J.-C. Wallet, Phys. Lett. A 224, 303 (1997); Y. Georgelin, T. Masson, and J.-C. Wallet, J. Phys. A 30, 5065 (1997).

${ }^{18}$ When the spins are not well split, it is a smaller group that is relevant, B.P. Dolan, Phys. Rev. B 62, 10278 (2000). 